\title{
The Stanford Hall coronavirus disease 2019 (COVID-19) remote rehabilitation assessment tool
}

\author{
Authors: Oliver O'Sullivan, ${ }^{\mathrm{A}}$ Robert Barker-Davies, ${ }^{\mathrm{A}}$ Michael Gough, ${ }^{\mathrm{A}}$ Sardar Bahadur, ${ }^{\mathrm{A}}$ Mark Cranley $^{\mathrm{A}}$ and \\ Rhodri Phillip ${ }^{\mathrm{A}}$
}

\section{Introduction}

Coronavirus disease 2019 (COVID-19) is a multisystem illness and can lead to significant morbidity that causes persistent symptoms and functional impairments. In order to assess sequalae and provide rehabilitation services to meet needs of individuals affected, a remote assessment tool was developed in May 2020 by Defence Medical Rehabilitation Centre (DMRC) Stanford Hall personnel.

\section{Materials and methods}

The tool was created by a multidisciplinary team (MDT) working group led by a rehabilitation consultant with a senior physiotherapist, occupational therapist, pain nurse consultant and exercise rehabilitation instructor. The draft version was reviewed and amended by the Clinical Delivery Group of the DMRC as an expert review panel and adjusted according to their feedback.

The tool was based on the current understanding of COVID-19 and experience of rehabilitation of other post-viral syndromes. It was designed to be performed over video-teleconference (VTC) platforms allowing the tool to be used immediately and consistently within the confines of the dynamic COVID-19 restrictions.

\section{Results and discussion}

The COVID-19 rehabilitation assessment tool incorporates a medical screening, identifying the acute course, severity and management of COVID-19. The impact of persistent postCOVID-19 symptoms was assessed including pain, fatigue, cognition, sleep and mood. Functional limitations, such as breathlessness, exercise intolerance, activities of daily living, and occupational considerations were also assessed. From this rehabilitation, key issues were identified and rehabilitation management plans were made.

In 5 months, 139 patients were assessed using the VTC tool. There were 167 consultations, with a range of patients who had acute illness severity ranging from community management to intensive care. The predominant symptoms included fatigue, shortness of breath on exertion and psychological sequalae. From those VTC assessments, 112 were recommended for 2-week residential rehabilitation. Twenty-eight patients required follow up to review. Twenty-six patients were deemed to have minimal rehabilitation needs and were provided with patient information resources.

\section{Conclusion}

Other, similar assessment methodologies are becoming more widely used, recognising the need to contact patients to explore prolonged impact of a post-COVID-19 syndrome. Patient feedback concerning the COVID-19 rehabilitation assessment tool has been positive, with an appreciation that enduring symptoms are to be expected, and that interventions are available to facilitate recovery. Further work is ongoing to consider optimal rehabilitation for postCOVID-19 symptoms.

\section{Conflicts of interest}

None declared.

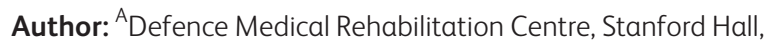
Loughborough, UK 